\title{
Suppressing R0 Requires the Identification of Super Spreader by Genetic Screening of Individuals without Fever
}

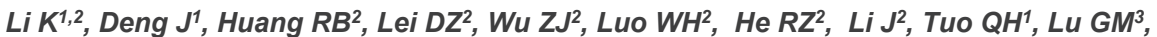 \\ Zhang $J^{2}$, Luo $D X^{2^{*}}$, Liao $D F^{1^{*}}$ \\ ${ }^{1}$ Division of Stem Cell Regulation and Application, Key Laboratory for Quality Evaluation of Bulk Herbs of Hunan \\ Province, Hunan University of Chinese Medicine, China \\ ${ }^{2}$ Translational Medicine Institute, The First People's Hospital of Chenzhou, Southern Medical University, China \\ ${ }^{3}$ Department of Medical Imaging, Jinling Hospital, Medical School of Nanjing University, China
}

\section{Keywords}

R0; Asymptomatic Spreader; SARS-CoV-2

\section{Short Communication}

The SARS-CoV-2 outbreak in Wuhan spreads naturally without official interventions before Mid-January of 2020, which allows the estimation of a relatively reliable R0 of SARS-CoV-2 being between 2.23 and $4.82[1,2]$. To efficiently fight against the worsening of an outbreak disease, administration intervention, including isolation of individuals infected or possibly infected, and screening of those who have had contact with a pathogen carrier or possible carrier. These interventions are aimed to suppress the R0 to smaller than 1 .

The SARS-CoV-2 infection has its own clinical characteristics: long incubation period (Figure 1), larger number of virus-carriers without clinical manifestation, and nearly half of pneumonia patients without fever and with negative genetic testing [3]. Accordingly, we ever suggested as early as on January 25 of 2020 to local and national offices about hospitalization of SARS-CoV-2 infected pneumonia without waiting genetic results and should be based on CT image (personal communication to Drs Liu and Tan).

Liao DF, Division of Stem Cell Regulation and Application, Key Laboratory for Quality Evaluation of Bulk Herbs of Hunan Province, Hunan University of Chinese Medicine,

Changsha 410208, Hunan, China, E-mail: dfliao@hnucm.edu.cn Received Date: 12 Mar 2020 Accepted Date: 26 Mar 2020 Published Date: 30 Mar 2020

Citation:

Li K, Deng J, Huang RB, Lei DZ, Wu $Z J$, Luo $W H$, et al. Suppressing RO Requires the Identification of Super Spreader by Genetic Screening of Individuals without Fever. Int J Fam

Med Prim Care. 2020; 1(2): 1006.

Copyright $\odot 2020$ Liao D. This is an open access article distributed under

the Creative Commons Attribution

License, which permits unrestricted use, distribution, and reproduction in any medium, provided the original work is properly cited.

As the outbreak has spread nationally and internationally, isolation of individuals infected or possibly infected may not be easily performed and also may not be effective as the difficulty in confirming the contact history epidemically. Screening super spreader without clinical manifestation is important as they are a risk subgroup with high R0 (Table 1). Therefore, we have the following suggestions and hope these suggestions will become routing strategies in helping China except Hubei province and the areas of other countries with the SARS-CoV-2 cases reported. We believe these strict genetic screening will efficiently suppress R0 to 1 or less.

1. Genetic testing of SARS-CoV-2 performed to individuals with contact history confirmed by

\begin{tabular}{ll}
\hline Figure 1: A chart graph showing the incubation period from 853 cases reported by 10 cities until February 11, \\
2020. \\
(*) The longest incubation period among the patients is 28 days.
\end{tabular}


Table 1: A collection of reported aggregation infection".

\begin{tabular}{|c|c|c|}
\hline Date & City & Number of infections \\
\hline $1 / 22 / 2020$ & ZheJiang NingBo & 11 \\
\hline $1 / 25 / 2020$ & GanSu LanZhou & 10 \\
\hline $1 / 25 / 2020$ & GanSu LanZhou & 7 \\
\hline $1 / 28 / 2020$ & AnHui HuangShan & 3 \\
\hline $1 / 29 / 2020$ & HuBei SuiZhou & 5 \\
\hline $1 / 30 / 2020$ & ZheJinag NingBo & 4 \\
\hline $2 / 1 / 2020$ & TianJin & 3 \\
\hline $2 / 3 / 2020$ & ZheJinag NingBo & 25 \\
\hline $2 / 3 / 2020$ & SiChuan NeiJiang & 3 \\
\hline $2 / 3 / 2020$ & BeiJing & 9 \\
\hline $2 / 3 / 2020$ & JiangSu NanJing & 2 \\
\hline $2 / 5 / 2020$ & AnHui HuaiAn & 7 \\
\hline $2 / 6 / 2020$ & GuiZhou QinNanZhou & 8 \\
\hline $2 / 6 / 2020$ & GuangDong DongGuan & 4 \\
\hline $2 / 6 / 2020$ & GuangDong ZhongShan & 3 \\
\hline $2 / 6 / 2020$ & GuangDong ZhongShan & 2 \\
\hline $2 / 6 / 2020$ & GuangDong ZhongShan & 2 \\
\hline $2 / 6 / 2020$ & GuangDong ZhongShan & 3 \\
\hline $2 / 6 / 2020$ & GuangDong FoShan & 4 \\
\hline $2 / 7 / 2020$ & GuiZhou ZunYi & 8 \\
\hline $2 / 8 / 2020$ & HuNsn LianYuan & 11 \\
\hline $2 / 9 / 2020$ & ZheJiang WenLing & 7 \\
\hline $2 / 9 / 2020$ & AnHui BengBu & 4 \\
\hline \multirow[t]{2}{*}{$2 / 9 / 2020$} & HuNan LouDi & 11 \\
\hline & Average of infections & 6.5 \\
\hline
\end{tabular}

(\#) Diamond Princess Cuise in Japanese has currently reported 174 individuals infected, which does not included as the final infected number is dramatically climbed daily.
CDC or CDC approved laboratories;

2. Officially reconstructing and confirming laboratories for genetic testing of SARS-CoV-2 to individuals without contact history;

3. Any person with positive results from 2 will be retested by CDC or CDC approved laboratory and following the isolation rule then;

4. The aforementioned strategies should be kept until R0 in the city or in the country is close to 0 , or local case is not reported in 2 to 3 week.

These suggestions should be carefully evaluated by WHO and performed as quick as possible. Before officially approval, we strongly believe that vehicles such as flight and cruise need to perform quick genetic screening as suggested above to avoid potential aggregation infection. The rule of universal precaution has already followed in the screening of potential HIV infection in blood donors. Since the new coronavirus outbreak has showed more severe risk to human health than SARS and MERS, genetic screening based on universal presentation as suggested, seems urgently needed.

\section{References}

1. Li Q, Guan X, Wu P, Wang X, Zhou L, Tong Y, et al. Early transmission dynamics in Wuhan, China, of novel coronavirus infected pneumonia. $\mathrm{N}$ Engl J Med. 2020.

2. Yang Y, Lu Q, Liu M, Wang Y, Zhang A, Jalali N, et al. Epidemiological and clinical features of the 2019 novel coronavirus outbreak in China. medRxiv. 2020.

3. Guan WJ, Ni ZY, Hu Y, liang WH, Ou CQ, He JX, et al. Clinical characteristics of 2019 novel coronavirus infection in China. medRxiv. 2020. 